\title{
Sanfilippo Disease
}

\author{
Barik KL', Kumar P², Ghosh TN³ ${ }^{3}$ De $S^{4}$, Basu $S^{5}$ \\ ${ }^{1}$ Dr. Kanai Lal Barik, MBBS, DCH, MD Associate Professor, ${ }^{2}$ Dr. Prabhat Kumar, MBBS, MDPG Trainee, ${ }^{3}$ Dr. Tarak Nath \\ Ghosh, MBBS, MD, Associate Professor, ${ }^{4}$ Dr. Sangita De, MBBS, PG Trainee, ${ }^{5}$ Dr. Samit Basu, MBBS, MDPG Trainee. All from \\ Department of Paediatrics, Burdwan Medical Hospital, Burdwan, India.
}

Address for correspondence: Dr. Prabhat Kumar, Email: prabhatbmch@gmail.com

\begin{abstract}
Sanfilippo disease is a type of Mucopolysaccharidosis, a hereditary progressive disease caused by mutation of gene for degradation of acid mucopolysaccharides. Early detection of this rare disease would enable screening and genetic counseling for asymptomatic family members.
\end{abstract}

Key word: Sanfilippo, Mucopolysaccharides

\section{Introduction}

Canfilippo disease (MPS 3) is a genetically heterogeneous but clinically similar group of 4 recognised types, each type is caused by a different enzyme deficiency involved in the degredation of heparin sulfate. Here a $6 \mathrm{yr}$ male child presented with hearing loss and abnormal behavior. On detailed work up it is nothing but a rare disease. In India it is a rare disease. Sanfilippo is diagnosed based on clinical features, radiographic results, urinary GAG screening test and finally definite diagnosis by Enzyme assay.

\section{The Case}

A six years male child presented with bilateral profound hearing loss, delayed milestones and abnormal behavior since the last four years. His developmental milestones up to two years of age was normal. Parents noticed gradual hearing loss, abnormal behavior also gradually increased and he lost acquired skills, visited different doctors and finally arrived to our OPD. There was no motor delay, no other family members had similar problem.

Patient was hyperactive, having aggressive destructive behavior ${ }^{6}$, temper tantrum and sleep disorder.

On examination-Facial abnormality were present; Dolichocephaly, depressed nasal bridge, broad nose, coarse hair, hirsutism. Anthropometry-wt 20 kg, ht-108 $\mathrm{cm}$, Upper to Lower segment Ratio being 1.05, Head circumference was $50 \mathrm{~cm}$ and Chest circumference was $61 \mathrm{~cm}$.

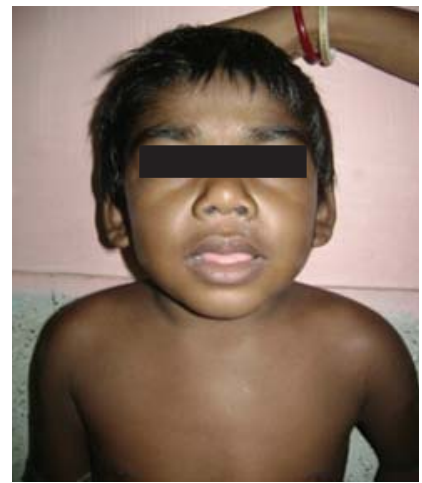

Fig 1: Showing Coarse facies, Dolichocephaly, depressed nasal bridge, broad nose, coarse hair, hirsutism in the patient.

BERA done shows profound hearing loss, I.Q $<75$ (below average), CT brain cortical atrophy with prominent cortical sulci and basal cistern, thyroid profile normal, and on fundoscopy there was no corneal clouding.

Table 1: Various Radiological Findings.

\begin{tabular}{|c|l|}
\hline X-Ray & \multicolumn{1}{|c|}{ Findings } \\
\hline Skull & Hyperostotic calvaria \\
\hline Wrist & $\begin{array}{l}\text { Proximal pointing of metacarpals with } \\
\text { bullet shaped phalanges, }\end{array}$ \\
\hline Chest & $\begin{array}{l}\text { Thickened medial } 3^{\text {rd }} \text { of clavicle with } \\
\text { spatulaed ribs,ovoid vertebral body }\end{array}$ \\
\hline Hip & Shallow acetabulum with flaring of ilius \\
\hline
\end{tabular}

Provisional diagnosis as MPS was kept in mind so urine for GAG was done which came positive. Definite diagnosis was done by enzyme assay; it confirmed Sanfilippo A. 



Fig 2: a) Shallow acetabulum b) Thickened medial $3^{\text {rd }}$ of clavicle and spatulated ribs ribs. c,d) Thick calvarium e) Bullet shaped phalanges $f$ ) Ovoid vertebral body

\section{Discussion}

Sanfilippo syndrome, or Mucopolysaccharidosis III (MPS-III) is a rare autosomal recessive ${ }^{2}$ lysosomal storage disease. It is caused by a deficiency in one of the enzymes needed to break down the glycosaminoglycan 'heparan sulfate $^{2}$ (which is found in the extra-cellular matrix and on cell surface glycoprotein).

The four types of MPS-III are due to specific enzyme deficiencies affecting the breakdown of heparan sulfate, which then builds up in various organs. MPS-III A, B, C and $D^{3,4,5}$ are considered to be clinically indistinguishable, although mutations in different genes are responsible for each disease.

The disease manifests in young children. Affected infants are apparently normal, although some mild facial dysmorphism may be noticeable. The stiff joints, hirsuteness and coarse hair typical of other mucopolysaccharidoses are usually not present until late in the disease. After an initial symptom-free interval, patients usually present with a slowing of development and/or behavioral problems, followed by progressive intellectual decline resulting in severe dementia and progressive motor disease ${ }^{6}$. Acquisition of speech is often slow and incomplete. The disease progresses to increasing behavioral disturbance including temper tantrums, hyperactivity, destructiveness, aggressive behaviour $^{6}$, pica and sleep disturbance. As affected children have normal muscle strength and mobility, the behavioral disturbances are very difficult to manage.
The disordered sleep in particular presents a significant problem to care providers. In the final phase of the illness, children become increasingly immobile and unresponsive, often require wheelchairs, and develop swallowing difficulties and seizures. The life-span of an affected child does not usually extend beyond late teens to early twenties.

Although the clinical features of the disease are mainly neurological, patients may also develop diarrhea, carious teeth, and an enlarged liver and spleen.

Of all the MPS diseases, MPS III produces the mildest physical abnormalities. It is important, however, that simple and treatable conditions such as ear infections and toothaches not be overlooked because of behavior problems that make examination difficult. Children with MPS III often have an increased tolerance of pain. Bumps and bruises or ear infections that would be painful for other children often go unnoticed in children with MPS III. Parents may need to search for a doctor with the patience and interest in treating a child with a long-term illness. Some children with MPS III may have a bloodclotting problem during and after surgery. The diagnosis may be confirmed by assay of enzyme levels in tissue samples and gene sequencing. Prenatal diagnosis is possible ${ }^{7}$.

Treatment remains largely supportive ${ }^{7}$ The behavioral disturbances of MPS-III respond poorly to medication. If an early diagnosis is made, bone marrow replacement may be beneficial. 
Along with many other lysosomal storage diseases, MPS-III exists as a model of a monogenetic disease involving the central nervous system. Several promising therapies are in development. Gene therapy is under investigation for MPS-III in animal models. Other potential therapies include chemical modification of deficient enzymes to allow them to penetrate the blood-brain barrier, stabilisation of abnormal but active enzyme to prevent its degradation, and implantation of stem cells strongly expressing the missing enzyme. For any future treatment to be successful, it must be administered as early as possible. Currently MPS-III is mainly diagnosed clinically, by which stage it is probably too late for any treatment to be very effective. Neonatal screening programs would provide the earliest possible diagnosis.

\section{Conclusion}

Early detection of this rare disease would enable screening and genetic counseling for asymptomatic family members. In future by gene therapy it is possible to produce enzyme which help in patients suffering from this disease.

\section{References}

1. Yano S, Moseley K, Pavlova Z. Postmortem studies on a patient with mucopolysaccharidosis type I: Histopathological findings after one year of enzyme replacement therapy. J Inherit Metab Dis. Mar 27 2009; [Epub ahead of print].
2. Zhang WM, Shi HP, Meng Y, Li BT, Qiu ZQ, Liu JT. [Postnatal and prenatal diagnosis of mucopolysaccharidosis type III (Sanfilippo syndrome)]. Zhonghua Er Ke Za Zhi 2008;46(6):40710.

3. Fan X, Tkachyova I, Sinha A, Rigat B, Mahuran D. Characterization of the biosynthesis, processing and kinetic mechanism of action of the enzyme deficient in mucopolysaccharidosis IIIC. PLoS One. 2011;6(9):e24951.

4. Ouesleti S, Brunel V, Ben Turkia H, Dranguet $H$, Miled A, Miladi N, et al. Molecular characterization of MPS IIIA, MPS IIIB and MPS IIIC in Tunisian patients. Clin Chim Acta. Nov 20 2011;412(23-24):2326-31.

5. Valstar MJ, Neijs S, Bruggenwirth HT, Olmer R, Ruijter GJ, Wevers RA, et al. Mucopolysaccharidosis type IIIA: clinical spectrum and genotype-phenotype correlations. Ann Neurol. Dec 2010;68(6):876-87.

6. Man TT, Tsai PS, Rau RH, et al. Children with mucopolysaccharidoses--three cases report. Acta Anaesthesiol Sin. Jun 1999;37(2):93-6.

7. Meikle PJ, Ranieri E, Simionsen $\mathrm{H}$, et al. Newborn screening for lysosomal storage disorders:clinical evaluation of a two-tier strategy. Pediatrics.2004;114 (4):909- 916.

\section{How to cite this article?}

Barik KL, Kumar P, Ghosh TN, De S, Basu S. Sanfilippo Disease. J Nepal Paediatr Soc 2012;32(3):263-265. 